\title{
Direct Numerical Simulation of Tollmien Schlichting Waves to Support Linear Stability Analysis
}

\author{
Heinrich Lüdeke, Viola Wartemann and Arne Seitz \\ DLR, Institute of Aerodynamics and Flow Technology, Braunschweig \\ Lilienthalplatz 7, D-38108 Braunschweig, Germany \\ heinrich.luedeke@dlr.de, viola.wartemann@dlr.de, \\ arne.seitz@dlr.de
}

\begin{abstract}
Summary
In the present study direct numerical simulations (DNS) of Tollmien-Schlichting waves in attached boundary layers will be shown in comparison with local linear stability theory (LST). The goal of the investigation is a simulation of such modes in separated flow where LST is limited due to its basic approach. The method provides an improved understanding of the physical mechanisms behind the transition scenario in the linear as well as in the non-linear regime.
\end{abstract}

\section{Introduction}

For present technical applications transition prediction in laminar boundary layers is mainly carried out by local and non-local Linear Stability Theory (LST) since alternatives like Direct Numerical Simulation (DNS) of complete configurations are computationally too expensive for realistic Reynolds numbers. Though proven as a reliable tool for aircraft design, LST techniques are restricted in different ways, for example their applicability in separated boundary layers is restricted by the underlying theory. A physically based simulation technique would consequently provide insight into the transition scenario in such complex flow fields and furthermore allow a verification of given LST methods.

Numerical studies of different accuracy and effort are possible, starting from stability calculations coupled with standard CFD simulations of entire configurations as well as basic studies of single forward-facing-step flow by DNS. All these techniques have to be verified by analytical solutions and validated by experimental data from literature.

Tollmien-Schlichting waves are well known as primary instabilities in the transition process of boundary layers from laminar to turbulent flow (Tollmien [9], Schlichting [7]). Since these modes are essentially two-dimensional instabilities their properties can be described by time accurate two-dimensional simulations, resolving the perturbation flow. For this purpose a $4^{\text {th }}$ order implicit finite difference method is chosen to resolve the modes of interest even at a limited grid resolution [1]. This method has shown very good results in the hypersonic regime by simulating Mack-modes in supersonic boundary layers [6]. 
In the following study validations between DNS and LST simulations of the 2D modes are discussed. They are shown, based on Blasius boundary layers as well as the profiles studied by Seitz [8] in comparison with results from the LST code NOLOT. Temporal approaches as well as spatial simulations by DNS are compared in the following sections with LST data of the growth rates and the perturbation profiles. Furthermore physical questions like saturation of instabilities are addressed.

\section{Numerical Approach}

\subsection{Direct numerical simulation}

The basic FLOWer code solves the compressible Reynolds-averaged Navier Stokes equations on block-structured grids with second order finite volume techniques. The high-order version, described in the following [1] uses fourth-order central differencing based on a standard compact approach. High-order compact filters that are applied at the end of each time step and sponge-zone boundary conditions are optional to reduce reflections. For the present work a $6^{\text {th }}$ order filter and the standard conservative form of the Euler terms is chosen. Time advancement is applied by a five-step second order Runge Kutta method. The solved equations are the compressible Navier-Stokes equations considering a perfect gas with density $\rho$, velocity components $u_{i}$, pressure $p$ and internal energy $e$, written in conservation law form as

$$
\begin{gathered}
\frac{\partial \rho}{\partial t}+\frac{\partial \rho u_{j}}{\partial x_{j}}=0 \\
\frac{\partial \rho u_{i}}{\partial t}+\frac{\partial \rho u_{i} u_{j}}{\partial x_{j}}+\frac{\partial p}{\partial x_{i}}=\frac{\partial \tau_{i j}}{\partial x_{j}}+f_{i} \\
\frac{\partial \rho E}{\partial t}+\frac{\partial(\rho E+p) u_{i}}{\partial x_{i}}=-\frac{\partial q_{i}}{\partial x_{i}}+\frac{\partial u_{i} \tau_{i j}}{\partial x_{j}}+g
\end{gathered}
$$

where $E=e+u_{i} u_{i} / 2$. Forcing terms $f_{i}$ and $g$ are included in the right hand side of the equations such that a specified parallel base flow $\bar{\rho}(y), \bar{u}_{i}(y), \bar{E}(y)$ is time independent for comparisons of DNS results with temporal LST data. In practice these terms are evaluated numerically within the code by computing and storing the initial residual. The equations are closed with the perfect gas law and constitutive relations for $q_{i}$ and $\tau_{i j}$ [6]. This approach is used in the following without modification for all grids using the described temporal approach as well as for spatial simulations without the source terms $f_{i}$ and $g$.

\subsection{Linear stability theory}

The NOLOT code [2][3], which is a spatial linear stability code, is a development of DLR and FOI and can be used for local as well as non-local analyses. In this work the local linear spatial approach is applied which is a subset of the non-local stability equations. The equations are derived from the equations of conservation of 
mass, momentum and energy, which governing the flow of a viscous, compressible, ideal gas, formulated in primitive variables. All flow and material quantities are decomposed into a steady laminar basic flow $\bar{q}$ and an unsteady disturbance flow $\tilde{q}$.

$$
q(x, y, z, t)=\bar{q}(x, y)+\tilde{q}(x, y, z, t)
$$

The disturbance $\tilde{q}$ is represented as a harmonic wave

$$
\tilde{q}(x, y, z, t)=\hat{q}(x, y) \exp [i(\alpha x+\beta z-\omega t)]
$$

with the complex-valued amplitude function $\hat{q}$. Since NOLOT is a spatial code the wave numbers $\alpha$ and $\beta$ are complex quantities and the frequency $\omega$ is a real value. $-\alpha_{i}$ is the complex growth rate. Since the chosen DNS approach is inherently temporal in nature, a Gaster transformation [6] of the LST growth rates is applied for comparisons between DNS and LST. The NOLOT code is validated by several test cases against published results, including DNS, PSE (parabolized stability equations), multiple scales methods and LST. A good summary of the validation cases is given by Hein et al.[2].

\section{Grids and Initial Solutions}

For the grid generation of the study a stretching function is used which provides an extended grid-point resolution near $y=0$, including geometries with a forward facing step. This wall normal grid distribution is given by a sinh function with an iteratively determined stretching factor. The temporal calculations use periodic boundary conditions in the $x$ and $z$ directions. No-slip walls at adiabatic wall temperature are applied. At the outer boundaries characteristic conditions are used. The initial solution for generic boundary layers and for inflow conditions is obtained from a separate solution of the similarity equations for compressible boundary layer flow, solved by a shooting method. Other cases are calculated for boundary layers from a NACA wing profile. For these cases a Mach number $M_{\infty}=0.204$, a Reynolds number based on the displacement thickness of $\mathrm{Re}=2900$, corresponding to flight No. 8, Measuring point 13 at $x / L=0.34$ from [8] is considered. The wall-normal co-ordinate as well as all other quantities derived from spatial co-ordinates are normalized by the displacement thickness. The temporal DNS of the instabilities is initialized by an artificial disturbance at $t=0$ which is given by an exponentially decaying harmonic function for the v-velocity component. This is a rough approximation of the eigenfunction which will decay during the first few thousand time steps while the amplified eigenfunction at the boundary layer will develop. For the spatial calculations the same technique is used at the inflow boundary with a temporal disturbance profile of the same shape. 


\section{Results}

\subsection{Validation of the temporal approach}

Pressure and velocity contours for the DNS calculations of Tollmien Schlichting waves are shown in Fig. 1 for the boundary layer flow with $M_{\infty}=0.204, \mathrm{Re}=$ 2900 to give an overview of the mode-shape in terms of these quantities. From these simulations perturbation profiles and amplification rates are extracted as shown in Fig. 2 in comparison with LST data, calculated by the NOLOT code. Due to the spatial LST calculations, the corresponding amplification rates are results of a Gaster transformation (Fig. 2b). Very good agreement is achieved for the eigenfunctions as well as for the amplification rates, which deviate by less than $0.4 \%$. To demonstrate the capabilities of the DNS approach the saturation process of a perturbed Blasius-flow is calculated for similar flow conditions but with a different $U_{\infty}$ at the boundary layer edge which reduces the growth rate in the linear regime of the transition process in comparison with the former calculation (see Fig. 3). In this figure the solid line is an extrapolation of the linear part while the squares are the ongoing DNS results. The saturation is clearly visible at $\ln (A)=-2$, which is a value within the expected regime of $1 \%$ of the boundary layer edge velocity (see [4]). The $\mathrm{v}$-component of the perturbation is shown in Fig. 3, bottom for time-instants from the linear (left) and non-linear regime (right). The influence of the saturation is especially visible in the near-wall region for the contour-lines of $v=0$. As a concluding remark, good agreement between the LST and DNS result is demonstrated.

\subsection{Spatial simulations}

To show the feasibility of DNS calculations for spatially developing instabilities with the described approach, a boundary layer profile over a flat plate is simulated. in analogy to the temporal approach only the wall normal component is perturbed at the inflow boundary by using the same approximation for the perturbation profile as for the respective temporal simulation. The chosen outflow condition is a simple first order extrapolation of the flow quantities. Five TS-wavelength are taken for the streamwise flowfield-extend with a resolution of about 16 cells per wavelength. A contour plot of the wall normal component in this flow field is shown in Fig. 4. The TS-waves show a visible growth in their amplitudes during their propagation in $\mathrm{x}$ direction, visible in the lower picture of Fig. 4, where the v-component is shown on the vertical axis. For such a small domain the growth of the perturbation is too small to calculate the amplification rate, so for a validation of the amplification factor with LST data, extended simulations are necessary.

With the described technique, simulations of the development of TS-waves over a forward facing step with re-circulating flow are carried out. A generic test case calculated by Kruse [5] with the DLR TAU code, is chosen. The flow conditions are $M=0.15, R e_{\text {hstep }}=1332$. A grid with 1536 streamwise cells and 360 spanwise cells is generated, scaled on the step height with $h_{\text {step }}=1$ and using the sinh-stretching function as already described. With this scaling the inflow plane is 
located at $x=-160$, the outflow plane at $x=+160$ and the farfield boundary at $y=44$. For the inflow condition a Blasius-profile with a displacement thickness of $\delta_{1, i n}=0.45 \cdot h_{\text {step }}$ (see [5]) is generated and perturbed in the already described way with an amplitude of $0.008 \cdot U_{\infty}$ and a frequency corresponding to a typical amplified wavelength behind the step of about $20 \cdot h_{\text {step }}$. This is a sufficiently large inflow-perturbation to guarantee the development of instabilities behind the step but results for the defined test case in TS-waves in the non-linear regime, so for comparison with LST data additional calculations with smaller amplitudes are necessary. The separation length in front of the step is approximately $3.8 \cdot h_{\text {step }}$ for the initial unpertturbed flow field, which is well in the range of the preliminary TAU-results (see Fig. 5). The resulting contours of the wall-normal velocity are shown in Fig. 6 for the perturbed flow in a selected region to demonstrate the mechanism of instability generation. While the wall-normal extend of the perturbations in front of the separation region becomes smaller, they are amplified again downstream of the reattachment. The reason for this behaviour can be seen in a harmonic perturbation of the separation by the incoming TS-waves and consequently a mode re-amplification downstream of the step. As expected the frequency of the re-amplified modes is the same as at the inflow plane and the wavelength is growing according to the growing boundary layer in this region. Additional simulations will have to confirm this hypothesis.

\section{Conclusion}

The present paper introduces a direct numerical simulation approach to study first modes in separated flow as a supporting technique for linear stability theory. The code is validated by the calculation of instability modes of different boundary layer profiles in comparison with LST data. For the shown investigated case good agreement between DNS and LST results in the linear regime can be demonstrated and the saturation process for the two-dimensional approach is simulated with good success. First spatial simulations of a Blasius boundary layer over a flat plate and the flow over a forward facing step have been carried out to show the feasibility of the approach in calculating such interesting cases. These simulations have to be continued in additional numerical studies.

\section{Acknowledgements}

Parts of this work were carried out while the first author was a Guest Scientist at the University of Southampton. The authors would like to acknowledge the support received from Prof. N. Sandham and Prof. Dr. C.-C. Rossow in setting up this visit. 


\section{References}

[1] S. Enk: "Ein Verfahren höherer Ordnung in FLOWer für LES". DLR IB-124-2007/8, Institut für Aerodynamik und Strömungstechnik, Braunschweig, Germany, 2007.

[2] S. Hein, F. P. Bertolotti, M. Simen, A. Hanifi, D. Henningson: 'Linear nonlocal instability analysis - the linear NOLOT code". DLR-IB 223-94 A56, 1994.

[3] S. Hein: "Linear and nonlinear nonlocal instability analysis for two-dimensional laminar separation bubbles". in: H.F. Fasel \& W. S. Saric (eds.), Laminar-Turbulent Transition, Proc. IUTAM Symposium on Laminar-Turbulent Transition, Sedona, Arizona, Sept. 1317, 1999, pp. 681-686, Springer, 2000.

[4] K.H. Horstmann, S.J. Miley: "Data report on flight and wind-tunnel investigations of Tollmien Schlichting Waves on an aircraft wing”. DLR IB-129-91/18, 1991.

[5] M. Kruse: Personal communication, 2010.

[6] N.D. Sandham, H. Lüdeke: "A numerical study of Mach 6 boundary layer stabilization by means of a porous surface". AIAA Aerospace Sciences Meeting 2009, Orlando USA, 2009.

[7] H. Schlichting: 'Zur Entstehung der Turbulenz bei der Plattenströmung”. Nachr. d. Ges. d. Wiss. zu Göttingen, Math.-Phys. Klasse, 1933, pp. 192-208.

[8] A. Seitz: "Freiflug-Experimente zum Übergang laminar-turbulent in einer Tragflügelgrenzschicht". Dissertation TU-Braunschweig, 2007, published as DLRForschungsbericht FB-2007-01.

[9] W. Tollmien: "Über die Entstehung der Turbulenz". Nachr. d. Ges. d. Wiss. zu Göttingen, Math.-Phys. Klasse, 1929, pp. 21-44.

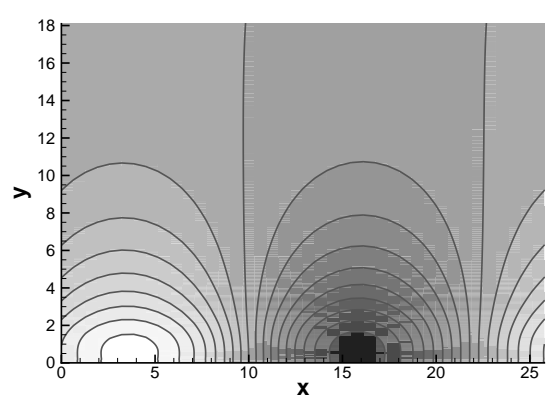

(a)

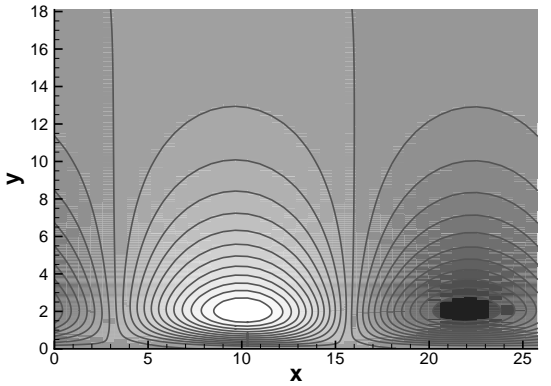

(b)

Figure 1: Tollmien Schlichting waves developing in a subsonic boundary layer at Re 2900 . (a) pressure contours, (b) contours of normal velocity 


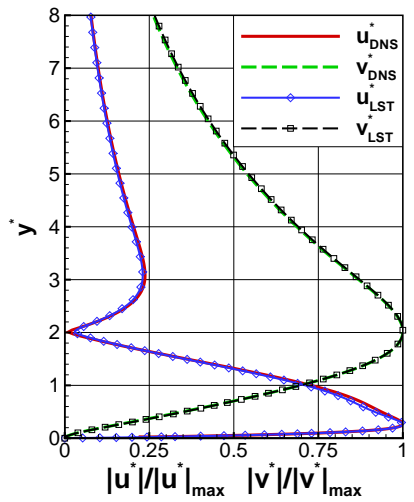

(a)

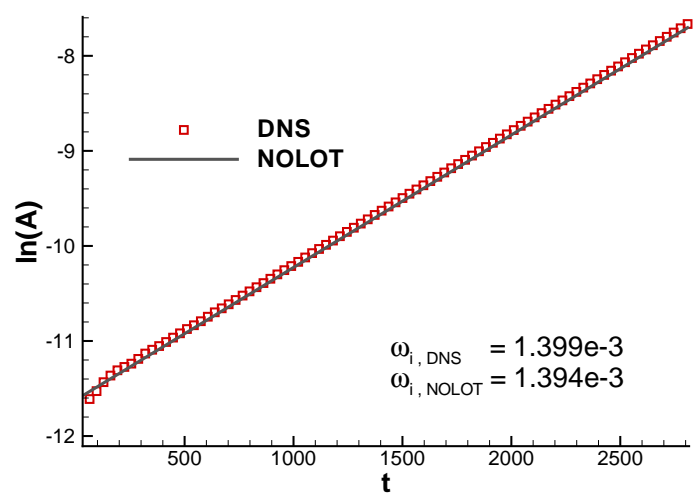

(b)

Figure 2: DNS and LST of a Tollmien Schlichting wave at $M=0.204, R e=2900$, $U_{\infty}=85.4 \mathrm{~m} / \mathrm{s}$, (a) comparison of eigenfunction shape. (b) Direct numerical simulation of disturbance amplitude (symbols) and linear fit of the growth rate (solid line)
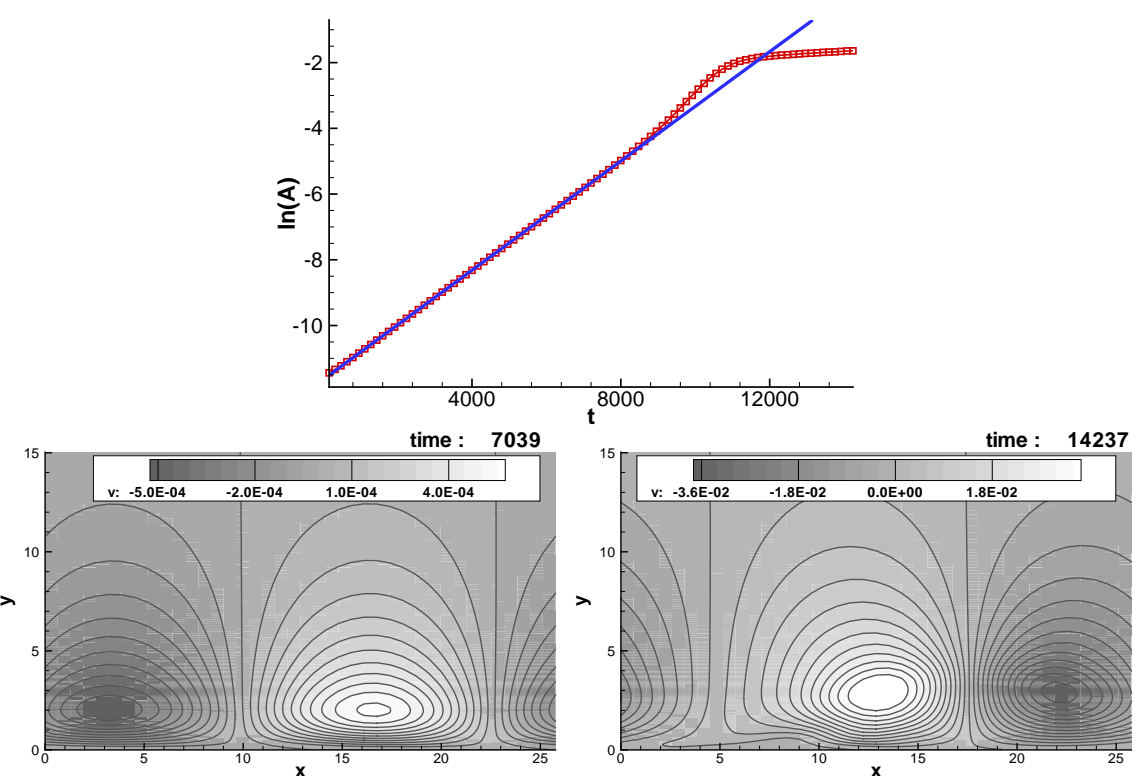

Figure 3: DNS and LST of a Tollmien Schlichting wave at $M=0.204, R e=2900, U_{\infty}=$ $66.7 \mathrm{~m} / \mathrm{s}$, Top: Direct numerical simulation of disturbance amplitude (symbols) and linear stability theory growth rate (solid line), bottom: v-contours of perturbation flow in the linear (left) and non-linear regime (right) 


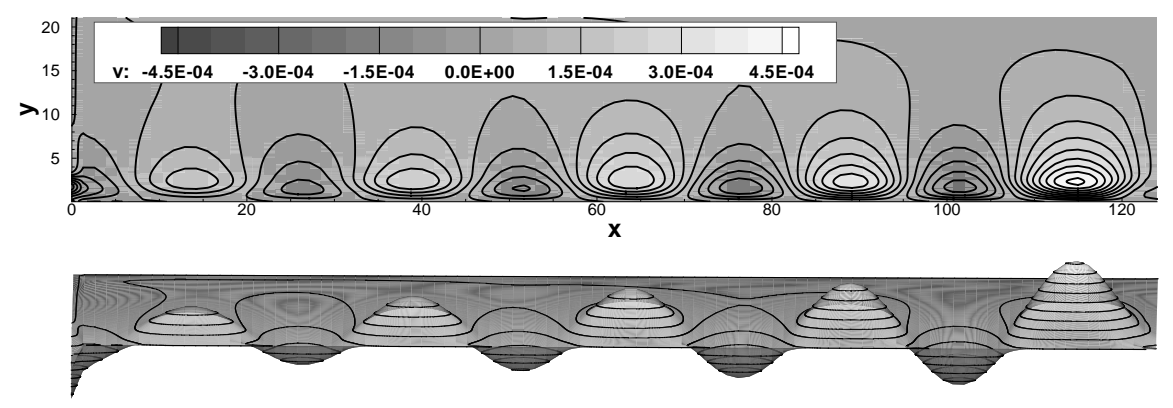

Figure 4: Spatial development of a Tollmien Schlichting wave developing in a subsonic boundary layer at $R e=2900$. Top: v-contours in the flow field, bottom: 3D view with wall normal component on vertical axis.

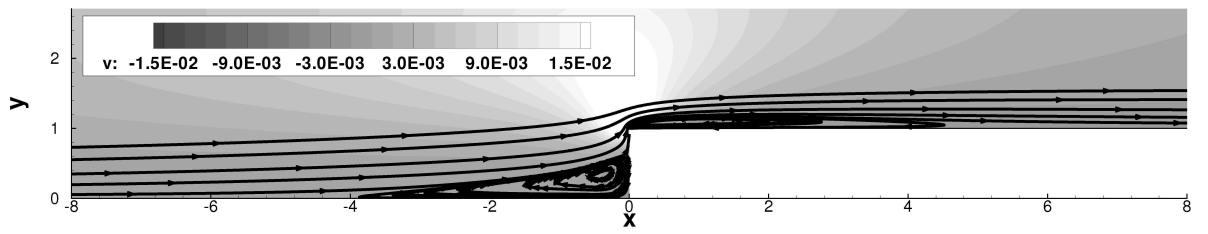

Figure 5: Seaparted base flow on a forward facing step at $M=0.15, R e_{h s t e p}=1332$.

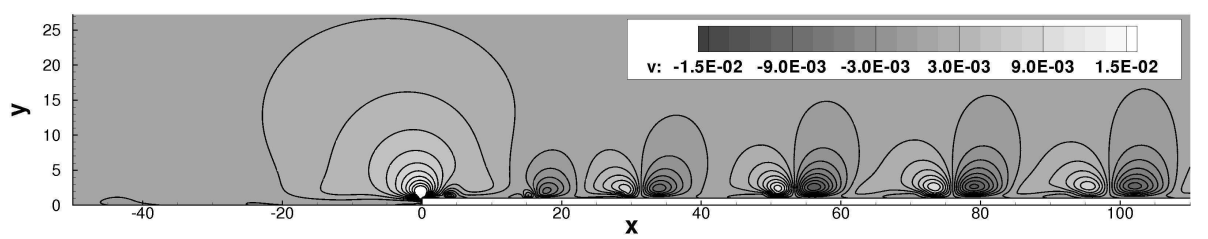

Figure 6: Spatial development of a Tollmien Schlichting wave over a forward facing step at $M=0.15, \operatorname{Re}_{\text {hstep }}=1332$. 